\title{
Structure, activation and biological effects of AKT or protein kinase B
}

Daniela MIRICESCU ${ }^{1}$, Alexandra TOTAN ${ }^{1}$, Constantin STEFANI ${ }^{2}$, Iulia-loana STĂNESCU ${ }^{1}$, Ana Maria Alexandra STANESCU ${ }^{3}$, loana Ruxandra RUSU ${ }^{4}$, Radu RADULESCU ${ }^{1}$, George Costin RUSU ${ }^{5}$, Maria GREABU ${ }^{1}$

${ }^{1}$ Department of Biochemistry, Faculty of Dental Medicine, "Carol Davila" University of Medicine and Pharmacy, Bucharest, Romania

2Department of Family Medicine, "Carol Davila" University of Medicine and Pharmacy, Bucharest, Romania

3"Carol Davila" University of Medicine and Pharmacy, Bucharest, Romania

${ }^{4}$ Department of Anatomy and Embryology, Faculty of Medicine,

"Carol Davila" University of Medicine and Pharmacy, Bucharest, Romania

${ }^{5}$ Department of Modern Languages, "Carol Davila" University of Medicine and Pharmacy, Bucharest, Romania

6"Carol Davila" University Central Emergency Military Hospital, Bucharest, Romania

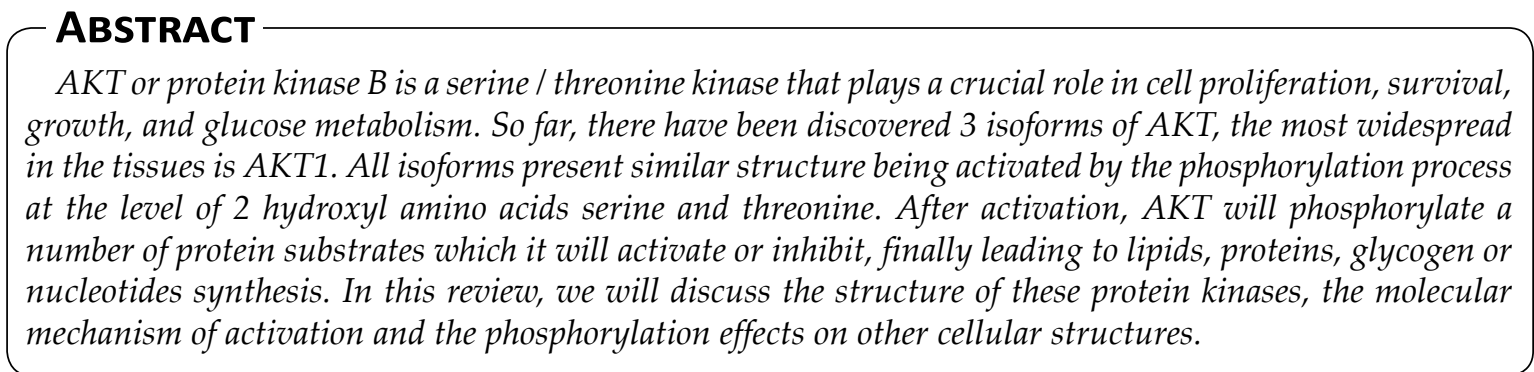

Keywords: protein kinase, phosphorylation, survival, proliferation

\section{AKT IDENTIFICATION AND STRUCTURE}

AKT or protein kinase $B$ belongs to the super protein kinase family named AGC after the kinases members, c-AMP-dependent protein kinase $A$ (PKA), protein kinase $G$ (PKG) and protein kinase $C$ (PKC). AGC kinases exhibited similar activation mechanisms and structural homology within the catalytic domain (1-3).

AKT is a serine / threonine kinase that was initially discovered in 1987 by Stefan Staal as the like- ly transforming gene component, v-AKT of AKT8 provirus. In the same study, Staal identified the human homologue of v-Akt, AKT 1 which was increased at patients with gastric adenocarcinomas (4).

In 1995, Richard Roth and co-workers discovered that AKT is activated by insulin (5). In mammals have been identified three AKT genes, termed AKT1/PKB $\alpha, A K T 2 / P K B \beta$ and the last one AKT3 / PKBŸ. Of all 3 isoforms, AKT 1 is the most widely distributed at the tissue level, being involved in 
cell growth and survival (6-8). AKT 2 is found at the muscular and adipocyte levels contributing to insulin mediated glucose homeostasis $(8,9)$. The last isoform, AKT 3, has been identified especially at brain and endocrine tissues (testes) levels (8-11).

All AKT izoforms have a highly conserved structure: an $\mathrm{N}$-terminal pleckstrin domain or $\mathrm{PH}$ domain, a kinase domain and a $\mathrm{C}$-terminal regulatory tail which contains the hydrophobic motif (11).

The N-terminal domain contains about 100 amino acids, which is similar to other protein kinases that binds 3-phosphoinositides and interacts with membrane lipid products such as phosphotidylinosiol 3,4,5 trisphosphate (PIP3) produced by phosphatidylinositol 3-kinase (PI3K) (11-13).

At catalytic domain level phosphorylation of the Thr residue occurs both in the case of PKB but also in PKA and PKC, thus leading to the partial activation of these protein kinases $(11,15,16)$.

The C-terminal domain contains about 40 amino acids having the following sequence in hydrophobic motif F-X-X-F / Y-Ser / Thr-Y / F, where X can be any amino acid. For the whole AGC protein kinases family phosphorylation of Ser and Thr residues is required for full activation in this hydrophobic motif (11). Akt 3 isoforms phosphorylate various substrates that contain in the $\mathrm{C}$-terminal region the following amino acid sequence: RXRXXSer /Thr, for exemple PRAS40 (proline-rich Akt substrate of $40 \mathrm{kDa}$ ) can be phosphorylated by all 3 isoforms but Akt 1 phosphorylates actin associated with palladin protein $(11,17)$.

\section{AKT ACTIVATION}

AKT signaling pathway is activated by various stimuli that are capable of inducing PIP3 formation by PI3K such as tyrosine kinase receptors, integrins, $T$ and $B$ cell receptors, cytokine receptors or receptors coupled with $\mathrm{G}$ proteins. In the extracellular domain of tyrosine kinase receptors (RTK), growth factors binds and will cause autophosphorylation of the receptor. Class 1 of PI3K binds to the phosphorylated receptor directly or via an adapter protein such as insulin receptor substrate 1/2 (IRS 1/IRS2).The PI3Ks will further catalyze phosphorylation of phosphatidyl inositol 4, 5 bisphostat ( PIP2) to PIP3 $(11,17)$.

PTEN (phosphate and tensin homology) performs dephosphorylation of PIP3 at PIP2. The interaction between AKT PH domain and 3-phosphoinositol induces a conformational change in AKT and PDK1 (3-phosphoinositide-dependent protein kinase 1) will phosphorylate Thr 308. For maximum activity, mTOR (mammalian target of rapamycin complex) will phosphorylate AKT Ser 473 from hydrofobic motif $(11,17,18)$.

Dephosphorylation of the 2 hydroxyl amino acids is carried out by PP2A (protein phosphatase 2A) specific Thr 308 and PHLPP (PH-domain leucine-rich-repeat-containing protein phosphatases) for Ser 473. Once activated Akt will dissociate from the membrane and further phosphorylate a wide variety of substrates, which are contained in the structure Ser or Thr such as protein or lipid kinases, transcriptional factors, metabolic enzymes $(11,17)$.

\section{AKT AND BIOLOGICAL EFFECTS}

Active AKT is involved in cell survival, growth and proliferation and glucose uptake as can be seen from Table 1.

The AKT / PKB signaling pathway plays a crucial role in regulating cell survival, helping the cells in the fight against apoptosis. Apoptosis is characterized in mammalian cells as an early process that is associated with the loss of mitochondrial integrity followed by the release of cytochrome $c$.

The cytochrome $c$ released then binds to the apoptotic protease-activating factor (Apaf -1) which it activates. Apaf- 1 binds and activates capase- 9 (proteases with cysteine residues), and initiates a caspase cascade, which are regulated by anti-apoptotic effectors ( $\mathrm{BCl}-2$ and $\mathrm{BCl}-\mathrm{xL}$ ) or pro-apoptotic proteins (Bad, Bid, Bik, Bax and Bak) $(19,20)$.

Bad is a member of the $\mathrm{BCl}-2$ protein family that is phosphorylated by AKT on Ser 136, so it no longer exhibits pro-apoptotic activity at the cell level and promotes cell survival $(20,21,22)$.

SAK (stress-activated protein kinase) is a family of protein kinases that regulates cellular response to stress or cytokines, consisting of 2 groups of kinases: JNK and p38 MAP kinases $(23,24)$.

ASK1 (apoptosis signal-regulating kinase) is a MAP kinase that usually induces apoptosis, will interact with AKT and is phosphorylated at Ser 83, thus inhibiting the apoptotic process and promoting cell survival. AKT will phosphorylate both MLK3 (mixed lineage kinase 3) on Ser 674 and SEK 1 on Ser 78, the activity of these kinases will also be inhibited as in the case of ASK1, promoting cell survival and not apoptosis $(25,26)$.

AKT promotes the regulation of cell survival through transcriptional factors that are responsible for pro and anti-apoptotic genes. The family of Fox or FH (forkhead) transcriptional factors has four Fox protein isoforms:Fox01, Fox02, Fox03, 
TABLE 1. The effects of AKT phosphorylation on different substrates (adapted from) (11).

\begin{tabular}{|l|l|l|l|}
\hline Cellular function & Substrate & Amino acid & AKT phosphorylation effect \\
\hline Cell survival & BAD & Ser 136 & Release of Bcl-2 proteins \\
& MLK3 & Ser 674 & Apoptosis inhibition \\
& ASK1 & Ser 83 & Apoptosis inhibition \\
& SEK1 & Ser 78 & Apoptosis inhibition \\
& FOX01 & Thr 24, Ser 256, 319 & Apoptosis inhibition \\
& FOX03 & Thr 32, Ser 253, 315 & Apoptosis inhibition \\
& FOX04 & Thr 28, Ser 139, 258 & Apoptosis inhibition \\
& MDM2 & Ser 133 & Inactivation of p53 \\
& Ik-B kinase & Thr 23 & Transcriptional activity of NF-kB \\
& CREB & Ser133 & Activation of antiapoptotic genes \\
& YAP & Ser 127 & Suppresor of apoptosis \\
\hline Cell growth & TSC complex & Ser 939,981, 1130, & Synthesis of proteins, lipids and \\
& (TSC1/TSC2) & 1132,Thr 11462 & nucleodites \\
\hline Glucose & GSK3 $\alpha$ & Ser 21 & Glycogen synthesis \\
homeostasis & GSK3 $\beta$ & Ser9 & Glycogen synthesis \\
\hline Cell proliferation & Cyclin D, & Thr 58, 286 & G1/S progression \\
& Cyclin B & Ser 354 & G2/M transition \\
\hline
\end{tabular}

Fox04 which can be directly phosphorylated by AKT. Phosphorylated Fox proteins promote cell survival through their action on specific target genes that normally inhibit cell survival $(27,28)$.

Family of nuclear transcription factor kB (NF$k B$ ) is a key regulator of immune response, and a deregulation of its activity leads to the development of pathologies such as autoimmune diseases and cancer $(29,30)$.

$\mathrm{NF}-\mathrm{kB}$ is activated by phosphorylation of the kinase complex IkB (inhibitor of kappa B kinases), which leads to its nuclear translocation and transcription of specific survival genes for $\mathrm{BCl}-\mathrm{xL}$ and caspase inhibitors (30-32).

Mdm2 (murine double minute) is an oncogene product induced by $\mathrm{p53}$, the major regulator of cell death in response to stress, especially when DNA damage occurs. AKT phosphorylates Mdm2 at 2 Ser residues, resulting in promoting inactivation or degradation of p53 and undermine the p53 to mediate pro-apoptotic transcriptional responses (33-35). CREB (Cyclic AMP (Camp)-response element binding protein) is a transcription factor, which can be phosphorylated by AKT on Ser 133, inducing expression of some antiapoptotic genes such as Bcl-2 (36).

YAP (Yes-associated protein) is phosphorylated by AKT on Ser 127 and in the phosphorylated form is a suppressor for apoptosis mediated by p73 transcriptional activity $(37,38)$.

AKT is involved in regulating cell growth through its effects on the tuberous sclerosis complex 1 and 2 (TSC1 / TSC2) and the mTORC signaling pathway. The primary mechanism by which AKT activates mTORC is the phosphorylation on Ser 2448 and TSC complex inhibition. TSC complex acts as a GAP specific for Ras-related GTPase Rheb, which will promote conversion of Rheb-GDP to Rheb-GTP and mTORC1 activation, which will further determine synthesis of proteins, lipids and nucleotides and autophagy (39). mTOR phosphorylates S6K1( kinases p70S6K1) and 4E-BP1(elF4E-binding protein 1), leading to increased translation and synthesis of cell-cycle-regulating and ribosomal proteins $(17,39)$.

AKT is involved in phosphorylation of glycogen synthase kinase 3 at the $\mathrm{N}$-terminus Ser residue, GSK-3 $\alpha$, Ser 21 and for GSK3 $\beta$, Ser 39. Phosphorylated GSK3, inhibits its kinase activity and also inhibits glycogen synthase. AKT-mediated inhibition of GSK3 activity, but dephosphorylates and activates glycogen synthase via PP1 (phosphoprotein phosphatase) which is activated by insulin or glucose leading to glycogen synthesis (17).

There is a close relationship between AKT and GSK3 in terms of metabolism and cell survival: phosphorylation and inbibation of GSK-3 mediates some of the effects of AKT. Phosphorylation of GSK-3 by AKT was considered to be a mechanism by which cell proliferation is also achieved $(11,17)$.

AKT can phosphorylates protein tyrosine phosphatase 1B (PTB1B), which prevents insulin receptor (IR) dephosphorylation and translocation of glucose transporter 4 (GLUT 4) from vesicular intracellular compartments to the plasma membrane and intracellular glucose uptake $(11,17)$.

AKT is involved in the control of the cell cycle beeing essential for meiosis, and dispensable for mitosis, by phosphorylating some target proteins that will lead to their activation or inactivation. AKT activetes cyclin B/CDK1 by phosphorylation, and coordinates the activation of cyclin $\mathrm{B} / \mathrm{cdk} 1$ 
(cyclin dependent kinase 1 ) at the centrosome and in the nucleus. Cyclins B, D,E are activated which will finally activate cdk2 and cdk1 which will determine G2 / M transition, cdk4 / 6 and chk2 will determine G1 / S transition (40).

\section{CONCLUSIONS}

AKT is a serine-threonine kinase that is activated by phosphorylation, which further phosphoryl- ates a number of proteins that contain Ser or Thr residues. These phosphorylations are essential for cell proliferation, growth and survival. In conclusion, the study of molecular mechanisms of AKT activation and further phosphorylation are crucial for a healthy human body.

\section{Acknowledgement}

All authors equally contributed to the present paper.

\section{Conflict of interest: none declared Financial support: none declared}

\section{REFERENCES}

1. Nicholson KM, Anderson NG.The Akt/PKB signalling pathway in human malignancy. Cell signal 2002; 14: 381-395.

2. Hajduch $E$, Litherland GJ, Hundal HS. Protein kinase B (Akt/PKB) - a key regulator of glucose transport? FEBS Lett 2001; 492: 199-203.

3. Huang X, Liu G, Guo J et al. The PI3K/AKT pathway in obesity and type 2 diabetes. Int J Biol Sci 2018; 14(11): 1483-1496.

4. Staal SP. Molecular cloning of the akt oncogene and its human homologues AKT1 and AKT2: amplification of AKT1 in a primary human gastric adenocarcinoma. Proc Natl Acad Sci. 1987 ;84(14):5034-7.

5. Kohn AD, Kovacina KS, Roth RA. Insulin stimulates the kinase activity of RAC-PK, a pleckstrin homology domain containing ser/ thr kinase. EMBO J 1995; 14(17):4288-95.

6. Chen WS, Xu PZ, Gottlob K et al. Growth retardation and increased apoptosis in mice with homozygous disruption of the Akt1 gene. Genes Dev 2001; 15 (17):2203-8.

7. Dennison KL, Robertson WR, Lewis BD et al. Functions of AKT1 and AKT2 potassium channels determined by studies of single and double mutants of Arabidopsis. Plant Physiol 2001; 127(3):1012-9.

8. Kitz A, Marcken M, Gautron AS et al. AKT isoforms modulate Th1-like treg generation and function in human autoimmune disease. EMBO 2019;20(8):e48624.

9. Garofalo RS, Orena SJ, Rafidi K et al. Severe diabetes, age-dependent loss of adipose tissue, and mild growth deficiency in mice lacking Akt2/PKB beta. $J$ Clin Invest 2003;112(2):197-208.

10. Yang ZZ, Tschopp O, Hemmings-Mieszczak $M$ et al. Protein kinase B/Akt1 regulates placental development and fetal growth. J Biol Chem 2003; 278 (34): 32124-32131.

11. Brown J, Banerji U. Maximising the potential of AKT inhibitors as anti-cancer treatments. PharmaThera 2017; 101-115.

12. Lietzke SE, Bose S, Cronin Tet al. Structural basis of 3-phosphoinositide recognition by pleckstrin homology domains. Mol Cell 2000; 6: 385-394.
13. Ferguson KM, Kavran JM, Sankaran VG et al. Structural basis for discrimination of 3-phosphoinositides by pleckstrin homology domains. Mol Cell 2000; 6: 373-384.

14. Jones PF, Jakubowicz T, Hemmings BA. Molecular cloning of a second form of rac protein kinase. Cell Regul 1991; 2:10011009.

15. Andjelkovic M, Jones PF, Grossniklaus U et al. Developmental regulation of expression and activity of multiple forms of the Drosophila RAC protein kinase. J Biol Chem 1995; 270:4066-4075.

16. Peterson RT, Schreiber SL. Kinase phosphorylation: Keeping it all in the family. Curr Biol 1999; 521-524.

17. Manning BD, Toker A. AKT/PKB signaling: Navigating the network. Cell 169 2017; 20:381-405.

18. Tang H, Tan X, Zhu L et al. Swimming prevents nonalcoholic fatty liver disease by reducing migration inhibitory factor through AKT suppression and autophagy activation. Am J Transl Res 2019;11(7):4315-4325.

19. Adams JM, Cory S.The Bcl-2 protein family: Arbiters of cell survival. Science 1998; 281 : 1322-1326.

20. Goan YG, Wu WT, Liu Cl et al. Involvement of mitochondrial dysfunction, Eedoplasmic reticulum stress, and the PI3K/AKT/mTOR pathway in nobiletin-induced apoptosis of human bladder cancer cells. Molecules 2019; 24(16).

21. Del Peso L, Gonzalez-Garcia M, Page C et al. Interleukin-3-induced phosphorylation of BAD through the protein kinase Akt. Science 1997; 278: 687-689.

22. Datta SR, Dudek H, Tao X et al. Akt phosphorylation of BAD couples survival signals to the cell-intrinsic death machinery. Cell 1997; 91: 231-241.

23. Johnson GL, Lapadat R. Mitogen-activated protein kinase pathways mediated by ERK, JNK, and p38 protein kinases. Science 2002; 298: 1911-1912.

24. Yeh YH, Liang CY, Chen ML et al. Apoptotic effects of hsian-tsao (Mesona procumbens Hemsley) on hepatic stellate cells mediatedby reactive oxygen species and ERK, JNK, and caspase-3 pathways. Food Sci Nutr 2019;7(5):1891-1898.

25. Barthwal MK, Sathyanarayana P, Kundu $\mathrm{CN}$ et al. Negative regulation of mixed lineage kinase 3 by Akt/PKB leads to cell survival. J Biol Chem 2003; 278: 38973902.

26. Park HS, Kim MS, Huh SH et al. Akt (protein kinase $\mathrm{B}$ ) negatively regulates SEK1 by means of protein phosphorylation. J Biol Chem 2002; 277: 2573-2578.

27. Burgering BM, Medema RH. Decisions on life and death: FOXO Forkhead transcription factors are in command when Akt/PKB is off duty. J Leukoc Biol 2003; 73: 689-701.

28. Feehan RP, Shantz LM. Negative regulation of the $\mathrm{FOXO3a}$ transcription factor by mTORC2 induces a pro-survival response following exposure to ultraviolet-B irradiation. Cell Signal 2016; 28(8):798-809.

29. Li Q, Verma IM. NF-KB regulation in the immune system. Nat Rev Immunol 2000; 2:725-734.

30. Ahmad R, Kochumon S, Chandy B et al. TNF- $\alpha$ Drives the CCL4 Expression in human monocytic cells: involvement of the SAPK/JNK and NF-KB signaling pathways. Cell Physiol Biochem 2019;52(4):908-921.

31. Barkett M, Gilmore TD. Control of apoptosis by Rel/NFkB transcription factors. Oncogene 1999, 18: 6910-6924.

32. Lauder A, Castellanos A, Weston K. C-Myb transcription is activated by protein kinase $B(P K B)$ following interleukin 2 stimulation of Tcells and is required for PKB-mediated protection from apoptosis. Mol Cell Biol 2001; 21: 5797-5805.

33. Singh S, Ramamoorthy M, Vaughan et al. Human oncoprotein MDM2 activates the Akt signaling pathway through an interaction with the repressor element-1 silencing transcription factor conferring a survival advantage to cancer cells. Cell Death Differ 2013; 20(4):558-566.

34. Gottlieb TM, Leal JF, Seger R et al. Cross-talk between Akt, p53 and Mdm2: 
possible implications for the regulation of apoptosis. Oncogene 2002; 21: 1299-1303.

35. Mayo LD, Donner DB. A

phosphatidylinositol 3kinase/Akt pathway promotes translocation of $\mathrm{Mdm} 2$ from the cytoplasm to the nucleus. Proc Natl Acad Sci 2001; 98: 11598-11603.

36. Li XY, Zhan XR, Liu XM et al. CREB is a regulatory target for the protein kinase Akt/ PKB in the differentiation of pancreatic ductal cells into islet $\beta$-cells mediated by hepatocyte growth factor. Biochem Biophys Res Commun 2011;404(2):711-6.

37. Wang C, Gu C, Jeong KJ et al. YAP/ TAZ-Mediated Upregulation of GAB2 leads to increased sensitivity to growth factor-induced activation of the PI3K Pathway. Cancer Res 2017; 77(7):16371648.

38. Basu S, Totty NF, Irwin MS et al. Akt phosphorylates the Yes-associated protein, YAP, to induce interaction with 14-3-3 and attenuation of p73-mediated apoptosis. Mol. Cell 2003; 11:11-23.

39. Saxton RA, Sabatini DM. mTOR signaling in growth, metabolism and disease. Cell 168 2017; 960-976.

40. Xu N, Lao Y, Zhang Y, et al. Akt: A double-edged sword in cell proliferation and genome stability. J Oncol 2012; 951724, 15 pages. 